\title{
Impacts des activités humaines sur les ressources forestières dans les terroirs villageois des communes de Glazoué et de Dassa-Zoumè au centre-Bénin
}

\author{
Brice TENTE ${ }^{1 *}$, Marcel Ayité BAGLO ${ }^{1}$, Jean C. DOSSOUMOU ${ }^{1}$ et \\ Hounnankpon YÉDOMONHAN ${ }^{2}$ \\ ${ }^{1}$ Laboratoire de Biogéographie et d'Expertise Environnementale, FLASH/UAC, BP526 Cotonou (Bénin). \\ ${ }^{2}$ Laboratoire de Botanique et d'Ecologie Végétale, FAST/ UAC, 01 BP 4521 Cotonou (Bénin). \\ *Auteur correspondant, E-mail : brice.tente@laposte.net ; Tél. (00229)95199977
}

\section{RESUME}

L'étude des impacts des activités anthropiques sur les ressources naturelles a été conduite dans les terroirs villageois des communes de Dassa et de Glazoué. Elle a été réalisée par le biais de l'analyse diachronique du couvert végétal entre 1998 et 2006, des relevés floristiques et des enquêtes socio-économiques sur la nature des pressions exercées par la population sur le couvert végétal. L'analyse de la dynamique des unités d'occupation du sol révèle une régression des formations naturelles au profit des formations anthropiques (champs et jachères). Les formations naturelles qui représentaient $27 \%$ des terroirs villageois en 1998 n'ont occupé que $20 \%$ de ces terroirs en 2006 et ne représenteront que $8,1 \%$ en 2025, si le taux actuel de dégradation $(0,45 \%)$ n'est pas freiné. Les peuplements ligneux des formations végétales actuelles sont caractérisés par une faible diversité spécifique. Leur densité est aussi faible (17-84 tiges/ha), sauf au niveau de la forêt claire/savane boisée où elle est de 276 tiges/ha. La surface terrière varie de $2,87 \mathrm{~m}^{2} / \mathrm{ha}$ au niveau des formations saxicoles à $11,08 \mathrm{~m}^{2} /$ ha dans la forêt-galerie. Les principales causes de la dégradation du couvert végétal sont la carbonisation, le prélèvement de bois et l'agriculture.

(c) 2011 International Formulae Group. All rights reserved.

Mots clés : ressources naturelles, dégradation, bois, activités agricoles.

\section{INTRODUCTION}

Le terroir villageois recouvre l'ensemble des zones d'activités villageoises (Gami, 1999), donc un espace naturel soumis en permanence à la pression humaine. Au Bénin, la mise en relation de l'évolution démographique et des besoins en terres cultivables pose le problème de la disponibilité en terre pour les générations à venir (Adam et Pofadji, 2008). Akoègninou et Akpagana (2001), ont montré que l'exploitation forestière, la transhumance, les feux de végétation et les techniques culturales prédatrices des espaces friches, constituent les principales causes de la perturbation de l'écosystème des terroirs villageois de la région des collines au centre-Bénin. Dans cette même partie centrale du Bénin, Oloukoï et al. (2006), ont fait remarquer que la probabilité pour qu'une unité d'occupation des terres soit transformée en champ ou en jachère dans les terroirs villageois est forte et varie de 30 à $53 \%$ sauf au niveau des agglomérations et des savanes saxicoles. 
Aussi, Yabi et al. (2008) ont-ils souligné que dans ladite région, l'environnement est assujetti, de plus en plus, à une ambiance d'agressivité climatique certaine, entraînant l'évolution négative inquiétante des composantes de l'environnement des terroirs villageois, malgré les mesures de conservation, prises par les mairies dans les Plans de Développement Communaux (PDC).

De nombreux travaux portant sur les ressources naturelles ont été conduits dans la zone d'étude. Ils ont surtout abordé l'étude de la flore et de la végétation (Adomou, 2005 ; Oumorou, 2003 ; Yédomonhan et al., 2008) et la modélisation de la dynamique de l'occupation des terres (Oloukoï et al., 2006). Des études corrélant la disponibilité et l'usage des ressources naturelles avec l'évolution démographique sont rares. Aussi, l'appréciation des changements d'états des unités d'occupation du sol a-t-elle été faite avec des modèles qui ne sont pas appropriés en science environnementale.

L'objectif global de la présente étude est d'identifier les formes d'usage des terroirs villageois et leurs impacts sur l'environnement.

\section{MATERIEL ET METHODE D'ETUDE Milieu d'étude}

La zone d'étude couvre deux communes (Dassa-Zoumè et de Glazoué) appartenant au Département des Collines (Figure 1). Sa superficie est de 334800 ha (Anonyme, 2001).

Le climat est de type tropical humide de transition et caractérisé par une température moyenne mensuelle de $28,04{ }^{\circ} \mathrm{C}$ et des précipitations moyennes mensuelles de $1067 \mathrm{~mm}$, avec 5 mois de saison sèche (Akoègninou et al., 2006).

La zone d'étude est située entièrement sur la pénéplaine cristalline du Moyen Bénin. Cette pénéplaine repose sur le vieux socle granito-gneissique précambrien, reliée au sud par les plateaux de terre de barre (Okioh, 1972 ; Anonyme, 1989). Elle est caractérisée par la présence de plusieurs collines en forme d'inselberg d'altitude pouvant atteindre 300 à 470 m et d'orientation générale N-S ou NNWSSE (Ahandjo, 2006; Yédomonhan et al., 2008).

Sur le plan pédologique, trois types de sols sont distingués dans la zone d'étude, à savoir : les sols ferrugineux, les sols minéraux bruts et les sols hydromorphes (Anonyme, 1989).

La végétation est une mosaïque de savanes et de forêts claires parsemées de forêts-galeries, de jachères, de champs et de plantations (Akoègninou et al., 2006). On y rencontre aussi des savanes et forêts denses sèches saxicoles (Yédomonhan et al., 2008).

La population compte 90475 et 93967 habitants respectivement à Glazoué et DassaZoumè (Anonyme, 2003). Leurs principales activités sont: l'agriculture, l'élevage, le commerce et l'exploitation forestière.

\section{Méthode}

La démarche méthodologique utilisée a consisté à réaliser une analyse diachronique du couvert végétal, des relevés floristiques et des enquêtes socio-économiques.

L'analyse diachronique du couvert végétal a été faite à l'aide des images satellites de 1998 et de 2006, provenant respectivement de LANDSAT TM et de ETM+. Les images ont été interprétées en utilisant les classes spectrales inspirées de la nomenclature à deux niveaux de classification (Oloukoï et al., 2006). La précision de la minute d'interprétation a été validée par le contrôle de terrain. Les logiciels utilisés sont Ilwis 3.2 pour l'interprétation des images et Arcview pour la réalisation des cartes d'occupation de sol. L'évolution des différentes unités d'occupation du sol a été déterminée de la façon suivante :

$$
\Delta_{\alpha}=\mathrm{S}_{2006}-\mathrm{S}_{1998} ; \text { où } \mathrm{S}_{1998} \text { est la }
$$
superficie occupée par l'unité $\alpha$ en 1998, $S_{2006}$ la superficie occupée par $\alpha$ en 2006 et $\Delta_{\alpha}$ exprime la variation de la superficie de l'unité $\alpha$ entre 1998 et 2006. 
Trois types d'évolution sont considérés : la stabilité $\left(\Delta_{\alpha}=0\right)$, l'évolution régressive $\left(\Delta_{\alpha}<0\right)$ et l'évolution progressive $\left(\Delta_{\alpha}>0\right)$. L'évolution des différentes occupations de sol a été modélisée et des projections d'occupations de sol sont faites à l'horizon 2025 pour ces unités suivant la méthode de Oloukoï et al. (2006). Le logiciel utilisé est Idrissi. La fonction utilisée pour l'allocation spatiale des probabilités prédites est CA_Markov dont l'algorithme est itératif afin de tenir compte des distances temporelles entre 1998 et 2006 (les deux dates d'analyse) et 2025 (la date de projection).

La carte de végétation établie à partir de l'image ETM+ de 2006 a servi au choix de trois zones de relevés forestiers. Ces zones ont été choisies de façon aléatoire mais de manière à couvrir tous les types de formations végétales. Dans chacune des zones, 31 placeaux de $30 \times 30 \mathrm{~m}^{2}$ ont été installés de façon systématique à tous les $100 \mathrm{~m}$. Les relevés forestiers n'ont pris en compte que les plantes ligneuses ayant un diamètre d'au moins $10 \mathrm{~cm}$ à hauteur de poitrine d'homme (d.b.h.) ou à $30 \mathrm{~cm}$ au-dessus des contreforts. Chaque plante ligneuse a été recensée et son d.b.h a été mesuré. Ces données collectées ont permis de déterminer la richesse spécifique, la surface terrière, la densité, l'indice de diversité de Shannon et l'équitabilité de Pielou pour chacune des formations végétales.

La richesse spécifique d'une formation végétale est le nombre d'espèces recensées. La surface terrière du peuplement est la somme des surfaces terrières de tous les arbres qui constituent le peuplement. Elle est obtenue par la formule :

$\mathrm{G}=(\pi / 4) \sum \mathrm{D}^{2}$, avec $\mathrm{G}=$ surface terrière exprimée en $\mathrm{m}^{2} /$ ha et $\mathrm{D}=$ diamètre à hauteur d'homme des arbres.

La densité des peuplements $(\mathrm{N})$ est le nombre de tiges par hectare. L'indice de diversité de Shannon est donné par la formule:

$\mathrm{H}=-\sum(\mathrm{Ni} / \mathrm{N}) \ln (\mathrm{Ni} / \mathrm{N})$, avec $\mathrm{Ni}=$ l'effectif de l'espèce i et $\mathrm{N}$, l'effectif total des espèces (Legendre et Legendre, 1984 ; Frontier et Pichod-Viale, 1991, 1995).

La diversité est faible lorsque $\mathrm{H}<3$ bits, moyenne si $\mathrm{H}$ est compris entre 3 et 4 puis élevée quand $\mathrm{H} \geq 4$ bits (Frontier et Pichod-viale, 1995). L'indice d'équitabilité de Pielou (E) est calculé suivant la formule : $\mathrm{E}=$ $\mathrm{H} / \mathrm{lnR}$, où $\mathrm{R}$ désigne la richesse spécifique.

Les enquêtes socio-économiques ont porté sur les rubriques suivantes: la nature des pressions exercées sur les ressources naturelles, les stratégies endogènes de gestion et leurs contraintes. Elles ont été réalisées par le biais de questionnaires suivant la méthode active de recherche participative (MARP). Les entretiens ont été semi-directs et directs. Le choix de l'échantillon a été fait de façon intentionnelle suivant les recommandations de Richard (1990). Au total, 312 personnes issues de différentes catégories socioprofessionnelles ont été enquêtées dans 33 villages dont 17 à Dassa et 16 à Glazoué. Ces villages ont été choisis dans les quatre zones retenues pour les relevés forestiers. L'échantillon est composé de 10 agents de l'administration forestière, 6 autorités des deux mairies, 2 agents des ONG impliquées dans les activités du Programme de Gestion des Forêts et Terroirs Riverains, 22 personnesressources capables de renseigner sur la dynamique de la végétation, 128 exploitants agricoles et 144 chefs de ménage agricole.

La projection de la population est faite sur une période allant de 2002 à 2025 et les taux d'accroissements utilisés sont de 4,30\% et $3,90 \%$, respectivement pour Glazoué et Dassa-Zoumè (Anonyme, 2003). La formule utilisée est :

$\mathrm{P}_{\mathrm{n}}=\mathrm{P}_{0}(\mathrm{t}+1)^{\mathrm{n}-\mathrm{no}} ;$ avec $\mathrm{P}_{\mathrm{n}}$, la population projetée à l'année $\mathrm{n} ; \mathrm{P}_{0}$, la population de 2002 ; t, le taux d'accroissement à l'échelle de la commune; n, l'année de projection et $\mathrm{n}_{0}$, l'année 2002 (Anonyme, 2003).

Le traitement et l'analyse des données statistiques et les tests de significativité sont faits avec les logiciels Excel 2007 et SPSS 12.0 . 


\section{RESULTATS}

Analyse diachronique de l'occupation du sol

La Figure 2 représente les cartes d'occupation du sol de 1998 (Figure 2a) et de 2006 (Figure 2b). Elles révèlent une tendance à l'expansion des agglomérations et des formations anthropiques au détriment des formations végétales naturelles. Les plus importantes régressions sont observées au niveau de la forêt claire/savane boisée et de la forêt-galerie avec respectivement $6,1 \%$ et $5,6 \%$ de taux de régression (Tableau 1). Par contre, la savane à emprise agricole est l'unité d'occupation qui a connu la plus forte expansion. Sa superficie passe de 24325 ha en 1998 à 32943 ha en 2006, soit un taux d'expansion de $3,8 \%$. Ces résultats témoignent de la forte dégradation des formations végétales dans les terroirs villageois considérés pour des fins agricoles.

Une simulation des tendances à l'horizon 2025 illustre une accentuation de la régression des formations naturelles, à savoir la forêt-galerie, la forêt dense, la forêt claire/savane boisée, la savane arborée et arbustive (Tableau 1). La superficie cumulée de ces formations naturelles qui est 69917 ha en 2006 passera à 28008,5 ha en 2025 . Ces formations naturelles ne représenteront alors que $8,1 \%$ de la superficie totale des terroirs villageois en 2025 , contre $20 \%$ en 2006 et $27 \%$ en 1998. La couverture forestière est passée de 13,6\% en 1998 à $10 \%$ en 2006, soit un taux annuel de déforestation de $0,45 \%$.

\section{Caractéristiques floristiques et structurales des peuplements ligneux actuels}

$\mathrm{La}$ richesse spécifique, l'indice de diversité de Shannon, l'équitabilité de Pielou, la densité et la surface terrière des peuplements ligneux des terroirs villageois de Dassa et de Glazoué sont consignés dans le Tableau 2.

La richesse spécifique des peuplements ligneux varie de 11 espèces dans la mosaïque de culture et jachère à 33 espèces dans les savanes arborée et arbustive. L'indice de Shannon est compris entre 1,9 bits et 2,5 bits. Ceci témoigne d'une faible diversité spécifique de ces habitats. L'équitabilité de Pielou varie de 0,7 à 0,8 ; elle traduit une bonne organisation des peuplements ligneux. La densité moyenne est comprise entre 17 et 276 tiges à l'hectare. La surface terrière est plus forte en forêt-galerie $\left(11,1 \% \mathrm{~m}^{2} / \mathrm{ha}\right)$ et plus faible dans la mosaïque de culture et de jachère $\left(1,9 \mathrm{~m}^{2} / \mathrm{h}\right)$.

Nature des pressions sur les ressources floristiques des terroirs villageois

Deux principaux types de pressions sont recensés : l'exploitation forestière et l'agriculture. Les types d'exploitations forestières sont, par ordre décroissant d'importance, la carbonisation, le prélèvement de bois de chauffe et le prélèvement de bois d'œuvre et de service. $96 \%$ des populations enquêtées utilisent le bois de chauffe pour les besoins en énergie de cuisson. De plus, la préparation de la boisson locale appelée "chakpalo", très prisée dans la localité, nécessite en moyenne $88 \mathrm{~m}^{3}$ de bois pour 100 litres de boisson. Compte tenu de son caractère commercial, la fabrication $\mathrm{du}$ charbon de bois prend une grande ampleur. Le charbon fabriqué est conditionné dans des sacs. Ces derniers sont exposés aux abords des routes (Figure 3) où ils sont achetés par les usagers de la route, surtout en direction des grandes villes comme Bohicon, Abomey, Abomey-Calavi et Cotonou.

En ce qui concerne l'exploitation agricole, c'est l'agriculture extensive qui est pratiquée. Cette technique est utilisée sans la mise en jachère des terres chez $90 \%$ des producteurs enquêtés. Seuls $10 \%$ des producteurs pratiquent la jachère. Les agriculteurs qui ne pratiquent pas la jachère estiment que cette technique n'est utilisable qu'en cas de manque d'espaces. Trois raisons principales sont évoquées par les producteurs pour l'extension des champs. $74 \%$ d'agriculteurs font les champs par extension des superficies pour augmenter leurs revenus monétaires, $18 \%$ pour avoir des sols toujours fertiles. Enfin, $8 \%$ des agriculteurs font l'agriculture extensive en réponse à l'augmentation de l'effectif de leurs ménages. 


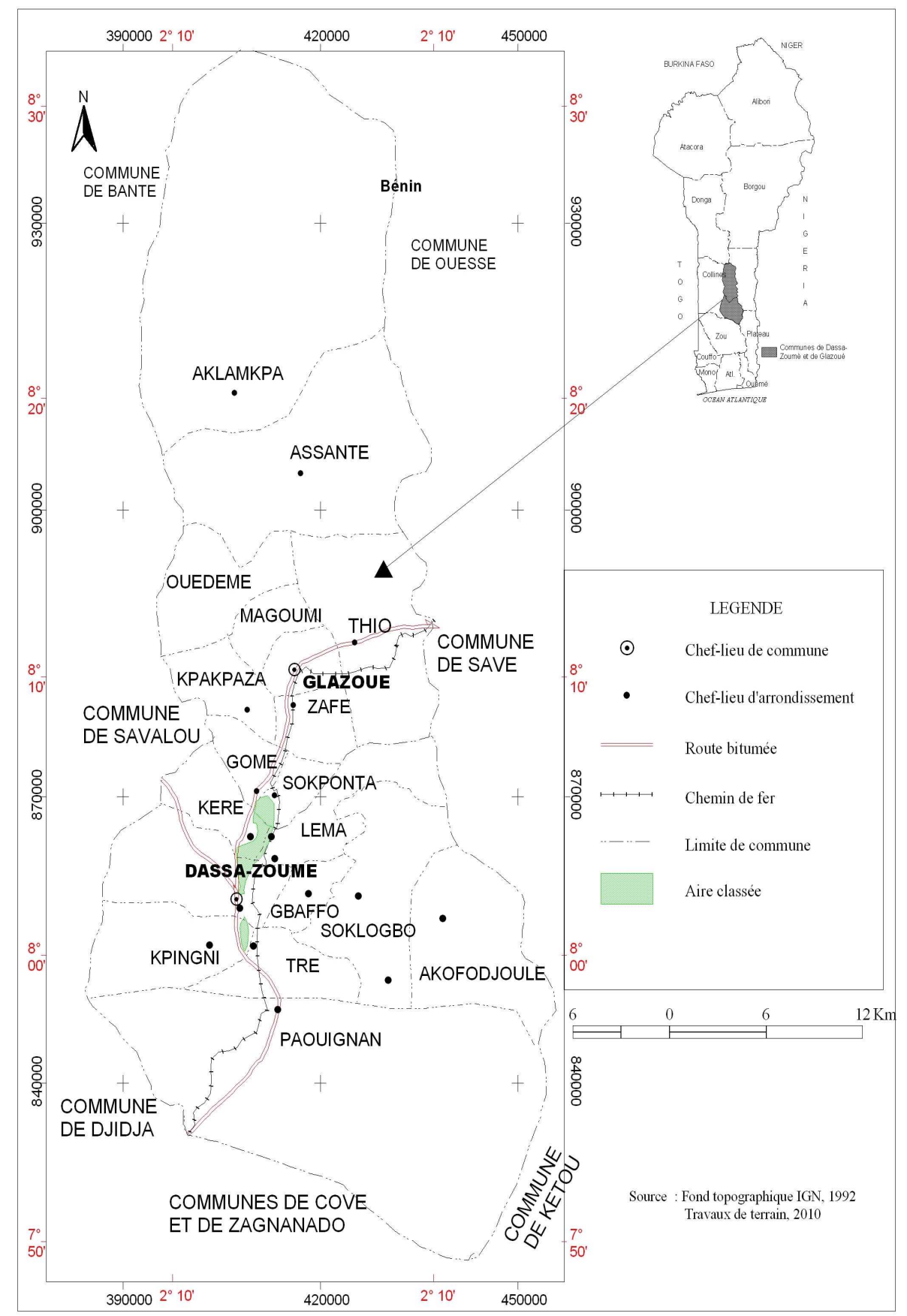

Figure 1 : Localisation de la zone d'étude. 

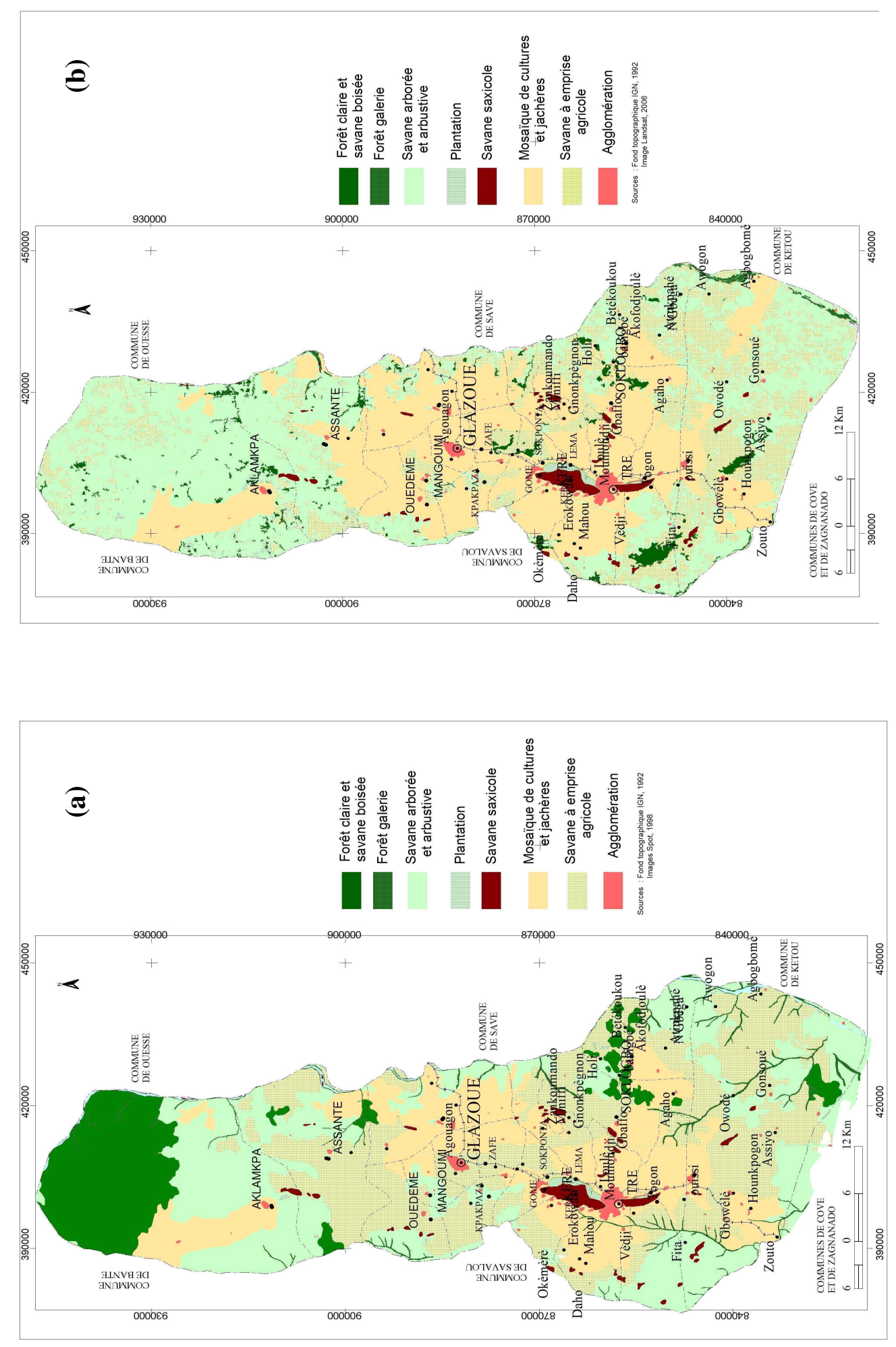

Figure 2 : Occupation des terroirs villageois de la zone d'étude en 1998 (a) et en 2006 (b). 


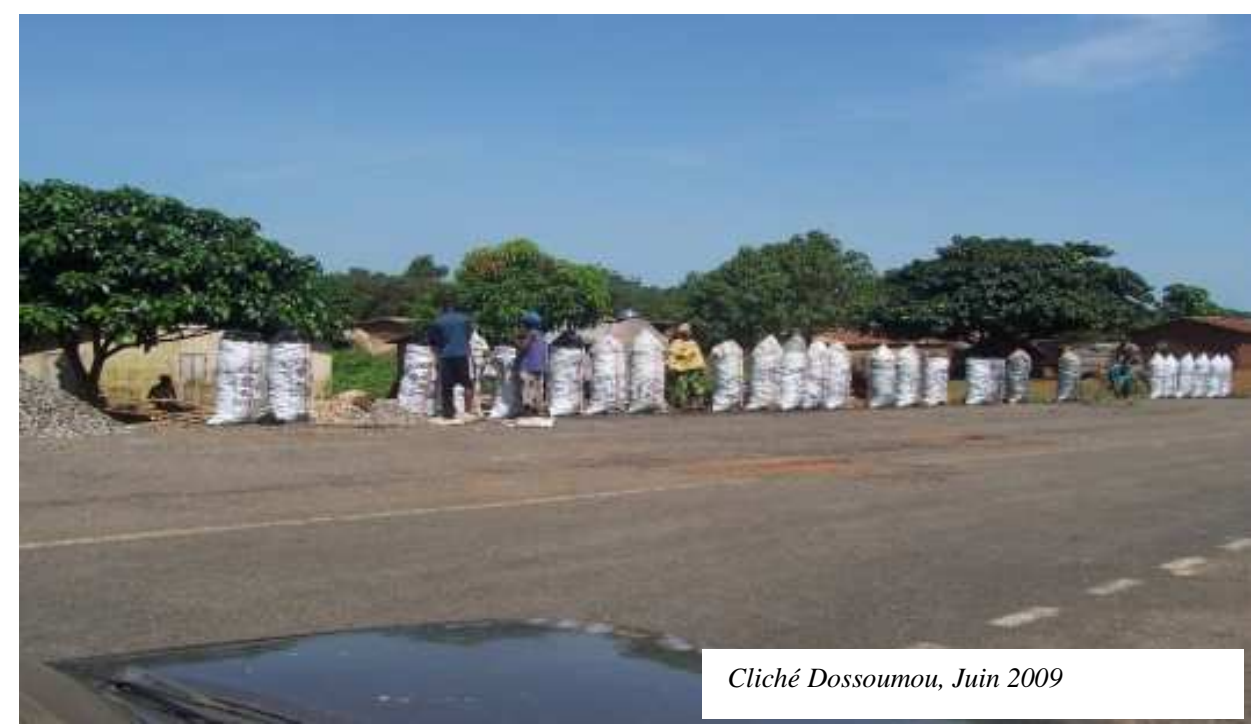

Figure 3 : Sacs de charbons de bois exposés au bord de la route bitumée à Zounto.

Tableau 1 : Taux d'expansion des unités d'occupation (1998 et $2006 ; 2006$ et 2025).

\begin{tabular}{lccccc}
\hline $\begin{array}{l}\text { Unité d'Occupation } \\
\text { du Sol }\end{array}$ & $\begin{array}{c}\text { Superficie } \\
\text { en 1998 } \\
\text { (ha) }\end{array}$ & $\begin{array}{c}\text { Superficie } \\
\text { en 2006 } \\
\text { (ha) }\end{array}$ & $\begin{array}{c}\text { S2006 - } \\
\text { S1998 }\end{array}$ & $\begin{array}{c}\text { Superficie } \\
\text { en 2025 (ha) }\end{array}$ & $\begin{array}{c}\text { S2025 - } \\
\text { S2006 }\end{array}$ \\
\hline Plantation & 8685,41 & 10772,5 & $\mathbf{0 , 6 0}$ & 15741,70 & $\mathbf{1 , 4 3}$ \\
Forêt-galerie & 4517,5 & 3023,25 & $\mathbf{- 0 , 4 3}$ & 521,25 & $\mathbf{- 1 , 0 3}$ \\
Forêt dense et saxicole & 210,59 & 173,75 & $\mathbf{0 , 0 0}$ & 0 & $\mathbf{0 , 0 0}$ \\
Forêt claire et Savane boisée & 34055 & 20850 & $\mathbf{- 3 , 8 1}$ & 10529,25 & $\mathbf{- 9 , 0 3}$ \\
Savane arborée arbustive & 55113,5 & 45870 & $\mathbf{- 2 , 6 6}$ & 16958 & $\mathbf{- 6 , 3 2}$ \\
Savane à emprise agricole & 24325 & 32943 & $\mathbf{2 , 4 8}$ & 46356,5 & $\mathbf{5 , 8 8}$ \\
Mosaïque de culture et Jachère & 217674 & 230740 & $\mathbf{3 , 7 6}$ & 253779,3 & $\mathbf{8 , 9 3}$ \\
Agglomération & 2919 & 3127,5 & $\mathbf{0 , 0 6}$ & 3614 & $\mathbf{0 , 1 4}$ \\
\hline
\end{tabular}

Tableau 2: Caractéristiques floristiques et structurales des peuplements ligneux actuels.

\begin{tabular}{|c|c|c|c|c|c|}
\hline Type d'occupation du sol & $\mathbf{R S}$ & $\begin{array}{c}\mathbf{H} \\
\text { (bits) }\end{array}$ & $\mathbf{E}$ & $\begin{array}{c}\mathrm{N} \\
\text { (tiges/ha) }\end{array}$ & $\begin{array}{c}\mathbf{G} \\
\left(\mathbf{m}^{2} / \mathbf{h a}\right)\end{array}$ \\
\hline Forêt-galerie & 15 & 2,23 & 0,82 & $76 \pm 25$ & $11,08 \pm 3,32$ \\
\hline Forêt claire/savane boisée & 17 & 2,43 & 0,86 & $276 \pm 127$ & $7,84 \pm 1,46$ \\
\hline Formation saxicole & 19 & 2,59 & 0,88 & $84 \pm 34$ & $2,87 \pm 0,66$ \\
\hline Savanes arborée/arbustive et à emprise agricole & 33 & 2,59 & 0,74 & $76 \pm 18$ & $6,15 \pm 0,28$ \\
\hline Mosaïque de Culture et Jachère & 11 & 1,98 & 0,82 & $17 \pm 14$ & $1,96 \pm 1,28$ \\
\hline
\end{tabular}




\section{DISCUSSION}

Les données collectées et analysées ont permis de dégager une tendance qui révèle l'augmentation des superficies agricoles, des savanes à emprise agricole et des agglomérations au détriment des autres catégories d'unité d'occupation du sol. Cette tendance confirme celles obtenues dans le Département des collines par Oloukoi et al. (2006), dans la préfecture du Zou par Allé (1998) et même sur le plateau d'Abomey par Vodougnon (1996). Cette dynamique est la conséquence de la pratique de l'agriculture itinérante sur brûlis comme principale technique culturale depuis des décennies dans ces terroirs villageois. A ces facteurs de pression, sont associés le prélèvement de bois de chauffe et la fabrication de charbon surtout pour des buts commerciaux.

Dans ce processus de forte pression sur les terroirs villageois, les risques de perturbation de l'équilibre écologique fragile de la zone d'étude ne sont pas épargnés. Akoègninou et Akpagana (2001) ont aussi signalé une expansion des champs et des jachères ainsi que des agglomérations, au détriment des formations forestières denses, conduisant à la raréfaction des grands arbres comme Afzelia africana, Anogeissus leiocarpa, Burkea africana, Pterocarpus erinaceus et Isoberlinia doka, utilisés comme combustible pour la fabrication de la farine de manioc.

L'analyse diachronique de l'occupation du sol de 1998 et de 2006, a permis de constater une perte de $0,5 \%$ de la couverture forestière par an. Ce taux est nettement supérieur au taux de $0,4 \%$ obtenu au plan national (Anonyme, 2001). Une des conséquences de cette dégradation est la migration des populations dans la conquête de nouvelles terres ou la mise en valeur des basfonds avec ses conséquences. Dans une étude plus approfondie au centre du Bénin, précisément dans les communes de Savè, de Ouèssè et de Tchaourou, Mama et al. (2001) ont constaté la même tendance. Ces auteurs ont remarqué que cette dynamique de l'occupation des terres affecte la cohésion sociale des populations, induit les populations à la conquête de nouvelles terres et déplace de plus en plus le front des activités agricoles des plateaux vers les milieux hydromorphes tels que les bas-fonds.

\section{Conclusion}

L'extension des terres cultivables et le prélèvement de bois-énergie constituent les principales formes de pression exercées sur les terroirs villageois des communes de Dassa et de Glazoué. Cette situation induit des modifications d'ordre floristique et structural du couvert végétal, avec une perte annuelle de $0,45 \%$ de la couverture forestière. La dynamique spatiale est régressive pour les formations végétales naturelles. Les savanes à emprise agricole, les mosaïques de cultures et jachères et les plantations constituent les unités d'occupation de sol qui ont connu d'extension avec un taux allant de 0,8 à $3,8 \%$. Cette évolution régressive pourrait entraîner un exode rural par suite de la pauvreté des sols et du manque de ressources forestières exploitables. Elle pourrait conduire aussi à l'insécurité foncière.

\section{REFERENCES}

Adam S, Pofadji M. 2008. Identification des espaces administratifs et de développement du Bénin: Approche géographique. Nouvelle Presse.

Adomou AC. 2005. Vegetation patterns and environmental gradients in Benin. Implications for biogeography and conservation. $\mathrm{PhD}$ thesis, Université de Wageningen, Pays-Bas, p. 136.

Ahandjo M. 2006. Milieu physique et promotion du tourisme dans la commune de Dassa-Zoumè. Mémoire de maîtrise, FLASH/UAC, Abomey-Calavi, p. 68.

Allé P. 1998. Dynamique des unités d'occupation du sol et contrainte foncière dans les sous-préfectures du Zou. Rapport d'activité, CENATEL, p. 87. 
Akoègninou A, Akpagana K. 2001. Etude cartographique et dynamique de la végétation de l'aire classée de la colline de Savalou (Bénin). Journal Botanique de la Société Botanique de France, 3 : 69-81.

Akoègninou A, van der Burg WJ, Maesen LJG. 2006. Flore Analytique du Bénin. Backhuys Publishers: Wageningen.

Anonyme. 1989. Notice explicative de la carte géologique à 1/200.000 - Feuille AbomeyZagnanado. Mém. 3, OBRGM, Cotonou, Bénin, p. 77 + carte.

Anonyme. 2001. Atlas monographique communes du Bénin. Ministère de l'Intérieur de la Sécurité et de la Décentralisation, Cotonou, Bénin.

Anonyme. 2003. Troisième recensement général de la population et de l'habitat. Direction des études démographiques. Ministère chargé du Plan, de la Perspective et du Développement. INSAE-UNICEF-UNFPA-DDC.

Frontier S, Pichod-Vitale D. 1991. Ecosystèmes : Structure, Fonctionnement, Evolution. Collection d'écologie 21, Masson: Paris (France).

Frontier S, Pichod-Vitale D. 1995. Ecosystèmes : Structure, Fonctionnement, Evolution ( $2^{\text {ème }}$ édition). Collection d'écologie, Masson: Paris (France).

Gami N. 1999. Les activités humaines dans les terroirs coutumiers, face aux plans d'aménagement des aires protégées: le cas du parc National d'Odzola, article scientifique, l'homme et la forêt tropicale, Congo, p.12.

Légendre L, Légendre P. 1984. Ecologie Numérique 2. La Structure des Données Ecologiques. Masson Collection d'Ecologie.
Mama V, Sokpon N, Sinsin B, Allé P. 2001. Rapport intégré sur l'état de l'environnement au Bénin. Rapport d'étude pour le MEHU.

Okioh L. 1972. Contribution à l'étude morphologique des reliefs résiduels de la région de Dassa-Zoumè, Fita et Savalou (Dahomey). Thèse du $3^{\text {è }}$ cycle de géographie, Université Paris, p. 170.

Oloukoï J, Mama V, Agbo F. 2006. Modélisation de la dynamique de l'occupation des terres dans le Département des Collines au Bénin. INRAB-Bénin/RECTAS-Nigéria, p. 8.

Oumorou M. 2003. Etudes écologique, floristique, phytogéographique et phytosociologique des inselbergs du Bénin. Thèse de doctorat unique en Sciences, Université Libre de Bruxelles, p. 210.

Richard JF. 1990. La Dégradation des Paysages en Afrique de l'Ouest. AUPELF, UICN, ORSTOM, ENDA, Presses Universitaires.

Vodougnon. 1996. Dynamique de l'environnement : cas du plateau d'Abomey. Mémoire de maîtrise de géographie, FLASH/UNB, p. 75.

Yabi F, Boko M, Afouda F. 2008. Quelques particularités de la variabilité pluviométrique dans un climat de transition : cas du département du Zou au Bénin, Afrique de l'ouest. Article scientifique, UAC/FLASH/LECREDE.

Yédomonhan $\mathrm{H}$, Houndagba $\mathrm{CJ}$, Akoègninou A, Maesen LJG. 2008. Structure et diversité floristique de la végétation des inselbergs du secteur méridional du Centre-Bénin. Syst. Geogr. Pl., 78: 111125. 